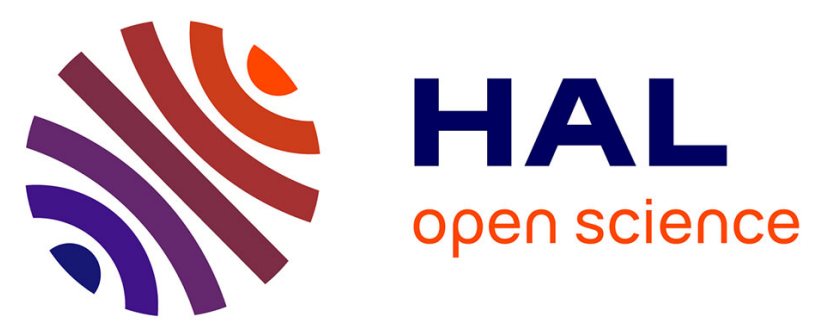

\title{
Thoracic NUT carcinoma: Common pathological features despite diversity of clinical presentations
}

\author{
Amal Fekkar, Camille Emprou, Christine Lefebvre, Gilbert Ferretti, Olivier
}

Stephanov, Daniel Pissaloux, Anne Mcleer, Anne-Claire Toffart, Sophie

Rousseaux, Saadi Khochbin, et al.

\section{To cite this version:}

Amal Fekkar, Camille Emprou, Christine Lefebvre, Gilbert Ferretti, Olivier Stephanov, et al.. Thoracic NUT carcinoma: Common pathological features despite diversity of clinical presentations. Lung Cancer, 2021, 10.1016/j.lungcan.2021.06.008 . hal-03364416

\author{
HAL Id: hal-03364416 \\ https://hal.science/hal-03364416
}

Submitted on 4 Oct 2021

HAL is a multi-disciplinary open access archive for the deposit and dissemination of scientific research documents, whether they are published or not. The documents may come from teaching and research institutions in France or abroad, or from public or private research centers.
L'archive ouverte pluridisciplinaire HAL, est destinée au dépôt et à la diffusion de documents scientifiques de niveau recherche, publiés ou non, émanant des établissements d'enseignement et de recherche français ou étrangers, des laboratoires publics ou privés. 


\section{Title}

2 Thoracic NUT carcinoma: common pathological features despite diversity of clinical

3 presentations

4

\section{Author list}

6 Amal FEKKAR ${ }^{1}$, Camille EMPROU ${ }^{1,2}$, Christine LEFEBVRE ${ }^{3}$, Gilbert FERRETTI ${ }^{4,5}$, Olivier

7 STEPHANOV $^{1}$, Daniel PISSALOUX ${ }^{6}$, Anne McLEER ${ }^{1,2}$, Anne-Claire TOFFART ${ }^{2,7}$, Sophie 8 ROUSSEAUX $^{2}$, Saadi KHOCHBIN ${ }^{2}$, Sylvie LANTUEJOUL ${ }^{5,6}$, Séverine VALMARY-

9 DEGANO ${ }^{1,2}$

11 (1) CHU Grenoble-Alpes, Department of pathology, 38043 Grenoble, France

12 (2) Institute for Advanced Biosciences, CNRS UMR5309, Inserm U1209, Grenoble Alpes 13 University, F-38000, Grenoble, France

14 (3) CHU Grenoble-Alpes, Laboratory of hematology,38043 Grenoble, France

15 (4) CHU Grenoble-Alpes, Department of radiology, 38043 Grenoble, France

16 (5) University of Grenoble Alpes, F-38000, Grenoble, France

17 (6) Centre Léon Bérard and CRCL, Department of biopathology, 28 rue Laënnec, 69373 Lyon, 18 France

19 (7) CHU Grenoble-Alpes, Department of Pneumology and Physiology, 38043 Grenoble, 20 France 


\section{ABSTRACT}

23 NUT carcinoma (NC), formerly known as NUT midline carcinoma, is a rare and very 24 aggressive cancer. It is genetically defined by the presence of acquired chromosomal rearrangement of the NUTM1 (NUclear protein in Testis Midline carcinoma family member 1) gene at chromosome $15 \mathrm{q} 14$ with a member of the bromodomain-containing protein (BRD) family gene, usually BRD4. Although primarily reported in the head and neck, and mediastinum locations of younger individuals, it is now established that $\mathrm{NC}$ arises in multiple sites in patients of all ages, with no gender predilection. NC is very likely to be underdiagnosed because of a lack of awareness of both clinicians and pathologists on the one hand, and of a nonspecific histological presentation on the other hand. As it is indistinguishable from other poorly differentiated carcinomas, pathologists should consider $\mathrm{NC}$ as a differential diagnosis of any poorly differentiated tumour. Diagnosis is now easily made by immunohistochemistry, using a highly sensitive and specific NUT monoclonal antibody. Despite chemo- or chemoradiotherapy, the prognosis of this tumour remains very poor. We report here a series of 3 cases of $\mathrm{NC}$ with different clinical and pathological presentations in order to draw attention on some common morphological features that can help clinicians and pathologists to think about this rare entity.

\section{$41 \quad$ Keywords}

NUT, carcinoma, BRD, thorax 


\section{INTRODUCTION}

NUT carcinoma (NC), formerly known as NUT midline carcinoma, is an aggressive, poorly differentiated subtype of carcinoma [1] defined by the presence of acquired chromosomal rearrangement of the NUTM1 gene (NUclear protein in Testis Midline carcinoma family member 1) localized on chromosome $15 \mathrm{q} 14$, with other genes including $B R D 4$ and, to a lesser extent, $B R D 3$ [2]. NC is underdiagnosed, mainly because of a nonspecific morphology, and its true incidence is therefore unknown [3]. NC was first described in the 1990s and has been reported primarily in the head and neck, and mediastinum of young individuals [4]. Nevertheless, it is now established that $\mathrm{NC}$ arises in multiple sites and affects patients of all ages, with no gender predilection [5]. NC is usually locally invasive and widely metastatic at diagnosis [6]. Despite therapy, median survival time is as low as 6.7 months and the estimated 2-year progression free survival is only of 9\% [6]. As NC resembles other poorly differentiated carcinomas, this diagnosis should be considered in case of poorly differentiated tumour [1] and verified by immunohistochemistry using a highly sensitive and specific NUT monoclonal antibody, which typically shows a speckled nuclear staining. Demonstration of a specific chromosomal rearrangement by molecular analyses such as RT-PCR or FISH [4] is no longer mandatory, even though FISH is commonly used to confirm the NUMT1 rearrangement.

With the aim of drawing attention to the common morphological features that can help pathologists to consider this rare diagnosis, we report 3 cases thoracic NC with diverse clinical presentations.

\section{Case report 1}

6 A 29-year-old female with no past history was admitted in 2010 for dyspnoea, haemoptysis and left supraclavicular pain. Thoracic, abdominal and pelvic computed tomography (CT) showed left mediastinal, left pulmonary and latero-uterine tumours with pleural and peritoneal 
effusions. Biopsy of the latero-uterine mass revealed an undifferentiated homogeneous proliferation, made of small-to-medium round malignant cells arranged in diffuse sheets. Nuclei harbour a vesicular chromatin and prominent nucleoli. A high mitotic rate was noted as well as many neutrophils within the stroma. At immunohistochemical analysis, tumour cells stained positively for CD45, CD43 and CD138 and were negative for epithelial markers, suggesting a haematological proliferation. The patient underwent chemotherapy with cytarabine and mitoxantrone. In the absence of any response to treatment, radical resection of the latero-uterine tumour was performed, whose analysis confirmed initial findings (Figure 1). Tumour karyotype showed a $\mathrm{t}(15 ; 19)$ translocation. Diagnosis of NC was therefore considered, and confirmed by immunohistochemistry with the NUT monoclonal antibody and by RT-PCR revealing a BRD4NUTM1 gene fusion. Despite chemotherapy with cisplatin and etoposide, the patient died within 2 weeks.

\section{Case report 2}

In 2018, a 19-year-old female presented with a 3 months history of neck pain with anorexia and weight loss. Contrast-enhanced CT showed a pathological fracture of $\mathrm{C} 4$ associated with a large pulmonary mass within the right lower lobe, extending to the inferior middle mediastinum (Figure 2) and many other lytic bone lesions. Microscopic examination of a biopsy performed on the left iliac wing showed an undifferentiated malignant tumour infiltrated by many neutrophils. The proliferation was composed of small to intermediate-sized cells with a monomorphic appearance and prominent nucleoli. Necrosis and high mitotic index were observed. Immunohistochemistry showed expression of cytokeratins, synaptophysin and epithelial membrane antigen by the tumour cells. In the absence of any clear diagnosis, NUT immunohistochemistry was performed, demonstrating a strong, speckled nuclear staining in $>80 \%$ of tumour cells. FISH showed a rearrangement of the NUTM1 gene in $80 \%$ of tumour 
cells. Despite chemotherapy (cisplatin and etoposide) and antalgic radiotherapy, the patient died less than 2 months after diagnosis.

\section{Case report 3}

A 51-year-old non-smoking male with no remarkable medical history presented in 2019 with a lung mass in the right lower lobe. Histological analysis showed a proliferation of poorly differentiated small to intermediate tumour cells. Many neutrophils infiltrated the tumour (Figure 3). Immunohistochemistry was positive for p40 and negative for TTF1, CD56, chromogranin and synaptophysin. PD-L1 showed a weak membranous positivity in $5 \%$ of tumour cells. ROS1 and ALK were negative. Neutrophilic infiltration of this poorly differentiated tumour was suggestive of the diagnosis of NUT carcinoma. Diagnosis was confirmed by immunohistochemistry and FISH. Despite chemotherapy with cisplatine and navelbine, the patient developed brain metastases and passed away 5 months after diagnosis.

\section{DISCUSSION}

Diagnosis of NUT carcinoma (NC) is difficult because this tumour lacks specific features in terms of risk factors or clinicopathological presentation. This case series suggests that in the presence of a poorly differentiated thoracic tumour, NC should be considered, especially when neutrophils infiltrate the tumour.

$\mathrm{NC}$ is a tumour reported to arise in various sites, with a predilection for the head and neck and mediastinum [7]. NC corresponds to up to $18 \%$ of all poorly differentiated carcinomas of the upper aerodigestive tract, particularly the sinonasal region [1]. Neither tobacco smoking nor viruses play a pathogenic role [1]. NC morphology is that of a poorly differentiated carcinoma, with a distinctly monomorphic, clonal appearance, displaying a focal squamous differentiation with abrupt keratinization in $33-40 \%$ of cases [1]. Tumour cells demonstrate frequent mitoses 
and single cell or geographic necrosis. The histologic features of $\mathrm{NC}$ are characteristic by the monomorphism of the cells, separation artefacts with a fried egg appearance with lack of

121 nuclear moulding, the abrupt squamous differentiation and, notably, the presence of infiltrating neutrophils [1]. In our 3 cases, the neutrophilic infiltrate was prominent and represents an important feature pointing towards this diagnosis. NC expresses with few exceptions cytokeratins and is usually diffusely positive for p63 antibody. Although rare, neuroendocrine markers and even TTF1 may be found positive and should not be used to rule out the diagnosis of $\mathrm{NC}$ and to favour the diagnosis of SCLC [1]. NC shows a positive immunostaining with the anti-BRG1 antibody, which makes it possible to exclude a deficient SMARCA4 / BRG1 tumour which is part of a novel group of undifferentiated tumours described in the latest WHO classification [8]. In 2009, the C52B1 monoclonal antibody raised against the NUT protein was

130 developed [1]. This antibody is highly specific, and does not stain other human tumours than 131 NC [2]. To be regarded as positive, NUT immunostaining must harbour a typical nuclear punctate pattern in more than $50 \%$ of tumour cells [9]. Using FISH as a "gold standard",

133 immunohistochemistry has a sensitivity of $87 \%$, a specificity of $100 \%$, a negative predictive 134 value of $99 \%$ and a positive predictive value of $100 \%$ [3]. FISH allows the detection of NUTM1 135 rearrangements but is unable to identify the fusion partner of NUTM1 [3]. For NUMT1 fusion detection by RT-PCR, specific probes have to be used. Most of the time, only BRD3- or BRD4-

137 NUT fusions are detected by RT-PCR [3]. RNA sequencing techniques allowing NUTM1 138 rearrangement detection without the knowledge of the partners could be interesting to discovery 139 of new or unusual fusions. Such an identification is relevant as a recent study indicates that 140 BRD3-NUT variants could be associated with a better survival than BRD4-NUT [5]. To date, 141 chemotherapy does not improve outcome of patients with NC [6]. However, a precise diagnosis 142 remains critical since experimental studies have revealed the molecular basis of the oncogenesis 143 induced by the BRD4-NUT fusion protein and identified new potential specific therapeutic 
144 strategies. NUT is a testis-specific factor and the recent generation of a mouse model where this 145 gene is invalidated has shown that NUT recruits p300 to induce a genome-wide histone 146 hyperacetylation which occurs in late differentiating male germ cells before histone-to147 protamine exchange [10]. In the case of BRD4-NUT fusion protein, NUT keeps its ability to 148 recruit $\mathrm{p} 300$, leading to the creation of hyperacetylated chromatin domains (BRD4-NUT foci)

149 [11]. Cellular CBP/p300 are sequestered in these foci, making them unavailable for the various 150 cell signalling involving these enzymes such as p53 acetylation in response to genotoxic 151 assaults [11]. Therefore, any therapeutic approach leading to the dispersion of these BRD4152 NUT foci could be considered as a valid therapeutic solution. With this regard, small molecules 153 directly targeting the BDR4 portion of BRD4-NUTM1, called bromodomain inhibitors, drive 154 terminal differentiation of NC cells in vitro and could thus inhibit BRD-NUTMI oncogene 155 expression [1,7]. Interestingly, among four patients with BRD4-NUTM1 fusions treated with the OTX015/MK-8628 bromodomain inhibitor, two of them had a rapid response with tumour regression and symptomatic relief, and one had relative tumour stabilization [1]. In addition, a

158 small molecule inhibitor of $\mathrm{CBP} / \mathrm{p} 300$ has been newly developed, which leads to a clear 159 dispersion of the BRD4-NUTM1 and p300 foci [11]. Based on the fact that the expression of 160 BRD4-NUTM1 is associated with globally decreased histone acetylation, histone deacetylase 161 inhibitors (HDACi) could also represent promising targeted therapeutic agents by restoring 162 chromatin acetylation and hence dispersing the oncogenic BRD4-NUT foci [1]. These 163 promising treatments reinforce the importance to identify $\mathrm{NC}$ among other undifferentiated 164 neoplasms especially thoracic SMARCA4-deficient undifferentiated tumours, which are poorly 165 sensitive to cytotoxic chemotherapy regimen and which unfortunately do not have a targeted 166 therapy [8].

167 In conclusion, our observation suggests to consider the diagnosis of NUT carcinoma in patients 168 presenting with a poorly differentiated chest malignant tumour, especially in the presence of 
169 neutrophilic infiltration. The availability of a highly specific monoclonal antibody makes the

170 diagnosis easy to perform by immunohistochemistry. FISH remains the "gold standard" for 171 diagnosis, but does not allow the identification of the fusion partner. RNA based assays such

172 as RT-PCR or RNA sequencing can allow the detection of the fusions transcripts provided they

173 suitably designed. Prognosis remains extremely poor, but targeted therapies seem promising in 174 patients harbouring BRD4-NUTM1 variants.

175

176 Acknowledgements

177 We thank Prof. Bruno Degano for critically editing the manuscript and Dr. Houda Kaddioui for 178 her help in implementing the figures.

179 
182 Figure 1. (A) The right radical adnexectomy leads to a tumour resection showing a diffuse

183 growth of poorly cohesive intermediate to large sized tumour cells with irregular vesicular

184 nuclei and prominent nucleolus. Many neutrophils infiltrate the tumour. (B) NUT monoclonal

185 antibody showing a speckled nuclear immunohistochemical staining. (C) R banded karyotype

186 after $24 \mathrm{~h}$ unstimulated culture performed on fresh ovarian tissue detected the specific 187 translocation $\mathrm{t}(15 ; 19)(\mathrm{q} 14 ; \mathrm{p} 13)$, associated with a translocation $\mathrm{t}(2 ; 12)(\mathrm{p} 23 ; \mathrm{p} 13)$ and other 188 structural abnormalities (16q, 20p). Red arrows: specific translocation t(15;19), small black 189 arrows: associated cytogenetic abnormalities.

191 Figure 2. (A) Axial contrast-enhanced CT images: A1- large heterogeneous soft tissue mass in 192 the right lower lobe extending in the inferior middle mediastinum (arrow). A2- Infiltrative 193 poorly circumscribed mass in left retropharyngeal space (arrows) associated with osteolysis of 194 the corpse of the 5th cervical vertebrae (arrow head) and extension within the cervical canal 195 (curved arrow). (B) Morphological aspects (Hematoxylin-Eosin-Saffron stain): nested cohesive 196 basophilic tumour cells with oval, hyperchromatic nuclei, a prominent nucleolus and small amounts of cytoplasm, with many neutrophils infiltrating the tumour. A giant osteoclastic cell

198 (arrow). Original magnification: x200 (B1), x400 (B2). (C) Immunohistochemistry: C1199 cytoplasmic expression of pancytokeratin AE1/AE3 antibody, x200. C2- cytoplasmic 200 expression of synaptophysin, x200. C3- NUT monoclonal antibody showing a speckled nuclear 201 immunohistochemical staining, $\mathrm{x} 400$.

203 Figure 3. A- Morphological aspects (Hematoxylin-Eosin-Saffron stain): diffuse growth of 204 anisokaryotic cells with round nuclei, prominent nucleoli and scanty, clarified cytoplasm. Many 
205 neutrophils infiltrate the tumour. x400. B- Immunohistochemistry: NUT monoclonal antibody 206 showing a speckled nuclear staining (immunoperoxydase, x400). C- Break apart Fluorescent In 207 Situ Hybridization (FISH) showing a NUTM1 gene rearrangement.

208 
210 [1] C.A. French, NUT Carcinoma: Clinicopathologic features, pathogenesis, and treatment: 211 NUT Carcinoma, Pathol Int. 68 (2018) 583-595. https://doi.org/10.1111/pin.12727.

212 [2] H. Haack, L.A. Johnson, C.J. Fry, K. Crosby, R.D. Polakiewicz, E.B. Stelow, S.-M. 213 Hong, B.E. Schwartz, M.J. Cameron, M.A. Rubin, M.C. Chang, J.C. Aster, C.A. French, 214 Diagnosis of NUT Midline Carcinoma Using a NUT-specific Monoclonal Antibody:, The 215 American Journal of Surgical Pathology. $33 \quad$ (2009) 984-991. 216 https://doi.org/10.1097/PAS.0b013e318198d666.

217 [3] C.A. French, NUT midline carcinoma, Cancer Genetics and Cytogenetics. 203 (2010) 218 16-20. https://doi.org/10.1016/j.cancergencyto.2010.06.007.

219 [4] A.G. Evans, C.A. French, M.J. Cameron, C.D.M. Fletcher, D.M. Jackman, C.S. Lathan, 220 L.M. Sholl, Pathologic Characteristics of NUT Midline Carcinoma Arising in the 221 Mediastinum:, The American Journal of Surgical Pathology. 36 (2012) 1222-1227. 222 https://doi.org/10.1097/PAS.0b013e318258f03b.

223 [5] N.G. Chau, C. Ma, K. Danga, H. Al-Sayegh, V. Nardi, R. Barrette, C.S. Lathan, S.G. 224 DuBois, R.I. Haddad, G.I. Shapiro, S.E. Sallan, A. Dhar, J.J. Nelson, C.A. French, An 225 Anatomical Site and Genetic-Based Prognostic Model for Patients With Nuclear Protein in 226 Testis (NUT) Midline Carcinoma: Analysis of 124 Patients, JNCI Cancer Spectrum. 4 (2020) 227 pkz094. https://doi.org/10.1093/jncics/pkz094.

228 [6] D.E. Bauer, C.M. Mitchell, K.M. Strait, C.S. Lathan, E.B. Stelow, S.C. Lüer, S. 229 Muhammed, A.G. Evans, L.M. Sholl, J. Rosai, E. Giraldi, R.P. Oakley, C. Rodriguez-Galindo, 230 W.B. London, S.E. Sallan, J.E. Bradner, C.A. French, Clinicopathologic Features and Long231 term Outcomes of NUT Midline Carcinoma, Clin Cancer Res. 18 (2012) 5773-5779. 232 https://doi.org/10.1158/1078-0432.CCR-12-1153. 
233 [7] L.M. Sholl, M. Nishino, S. Pokharel, M. Mino-Kenudson, C.A. French, P.A. Janne, C. 234 Lathan, Primary Pulmonary NUT Midline Carcinoma: Clinical, Radiographic, and Pathologic 235 Characterizations, Journal of Thoracic Oncology. $10 \quad$ (2015) 951-959. 236 https://doi.org/10.1097/JTO.0000000000000545.

237 [8] D. Lokuhetty, V.A. White, I.A. Cree, Thoracic Tumors, WHO Classification of 238 Tumours, 5th Edition, Volume 5, IARC Lyon, 2021.

239 [9] J.A. Bishop, C.A. French, S.Z. Ali, Cytopathologic features of NUT midline carcinoma: 240 A series of 26 specimens from 13 patients: Cytopathology of NUT Midline Carcinoma, Cancer 241 Cytopathology. 124 (2016) 901-908. https://doi.org/10.1002/cncy.21761.

242 [10] H. Shiota, S. Barral, T. Buchou, M. Tan, Y. Couté, G. Charbonnier, N. Reynoird, F. 243 Boussouar, M. Gérard, M. Zhu, L. Bargier, D. Puthier, F. Chuffart, E. Bourova-Flin, S. Picaud, 244 P. Filippakopoulos, A. Goudarzi, Z. Ibrahim, D. Panne, S. Rousseaux, Y. Zhao, S. Khochbin, 245 Nut Directs p300-Dependent, Genome-Wide H4 Hyperacetylation in Male Germ Cells, Cell 246 Reports. 24 (2018) 3477-3487.e6. https://doi.org/10.1016/j.celrep.2018.08.069.

247 [11] N. Reynoird, B.E. Schwartz, M. Delvecchio, K. Sadoul, D. Meyers, C. Mukherjee, C. 248 Caron, H. Kimura, S. Rousseaux, P.A. Cole, D. Panne, C.A. French, S. Khochbin, Oncogenesis 249 by sequestration of $\mathrm{CBP} / \mathrm{p} 300$ in transcriptionally inactive hyperacetylated chromatin domains, 250 EMBO J. 29 (2010) 2943-2952. https://doi.org/10.1038/emboj.2010.176. 


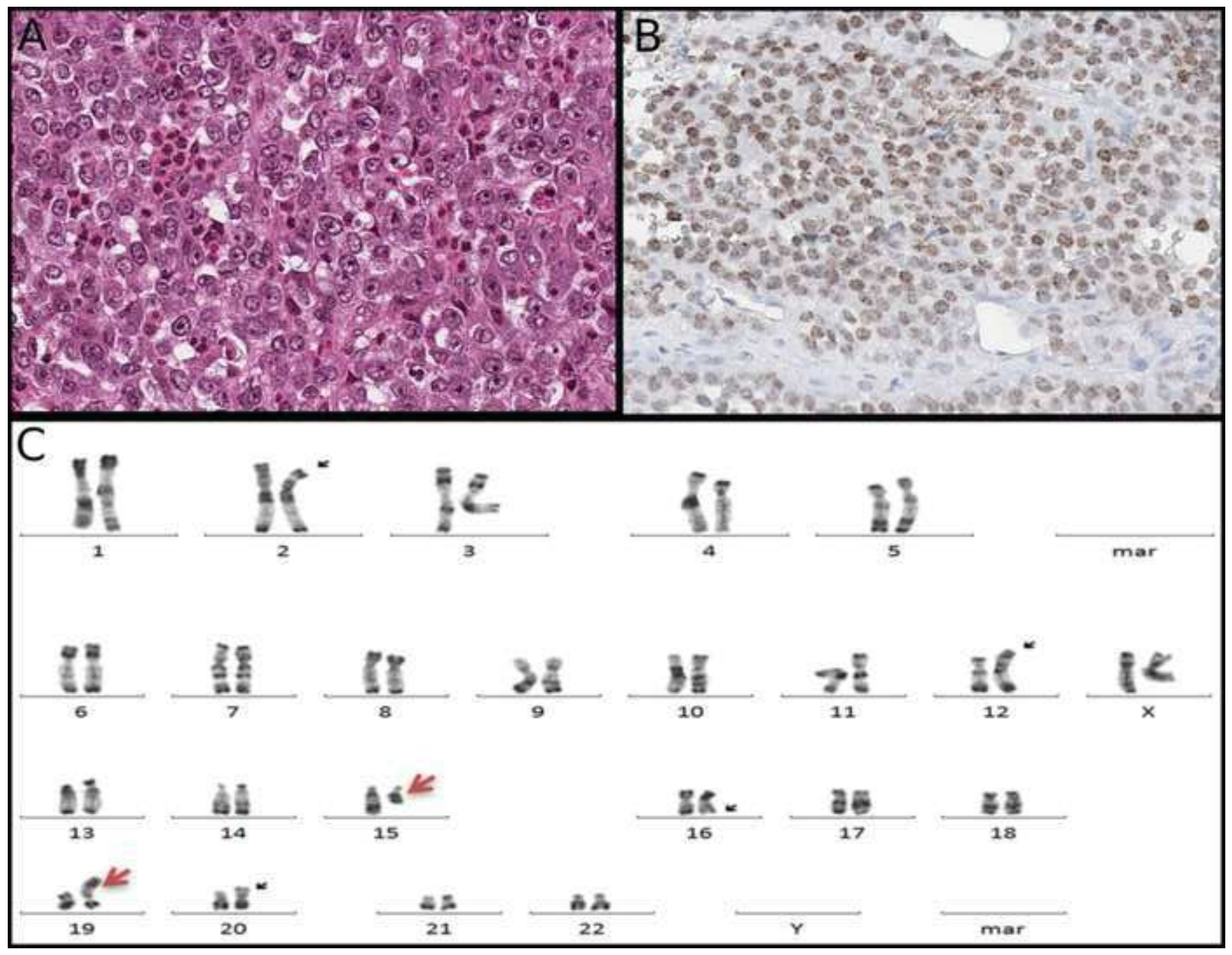



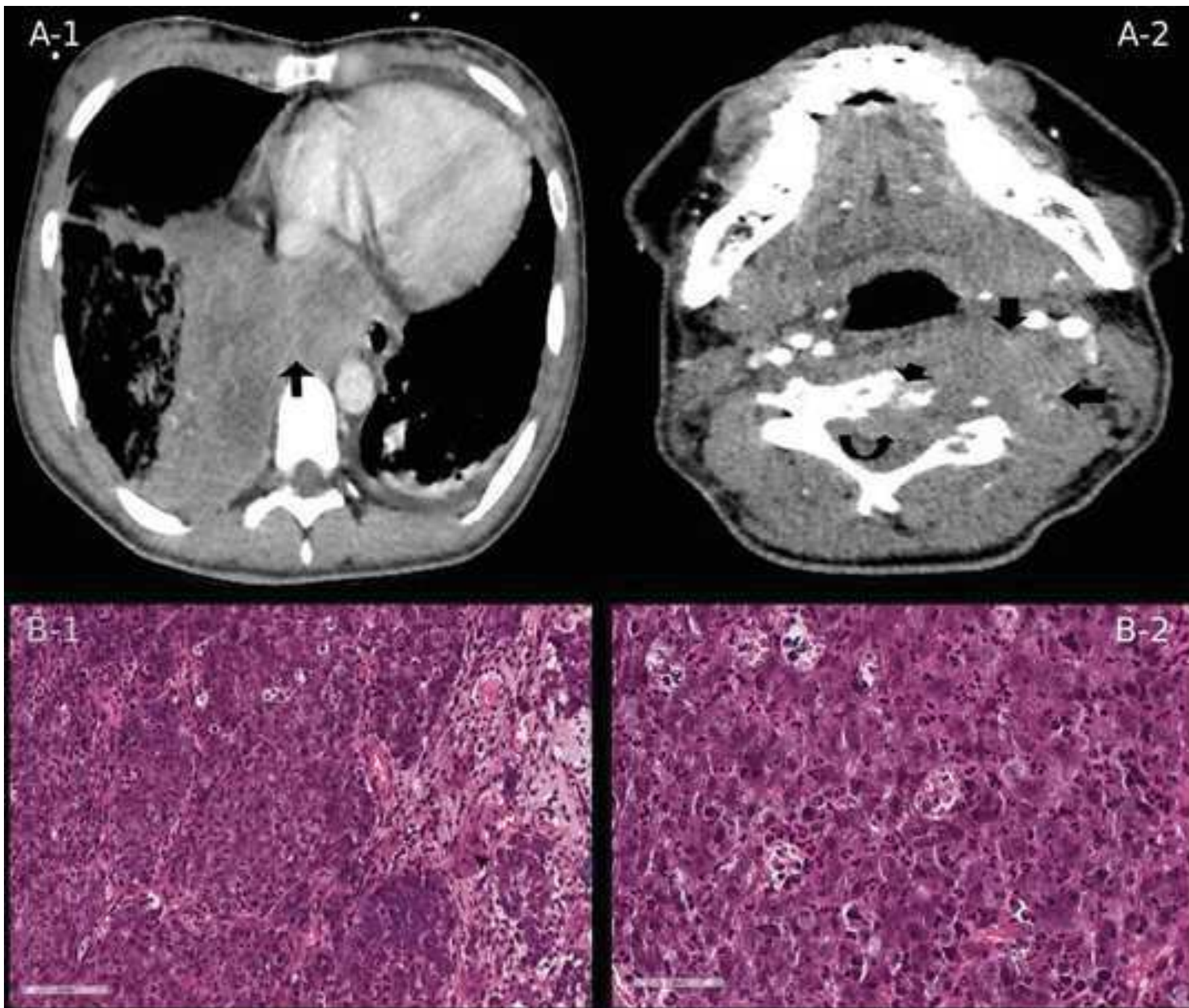

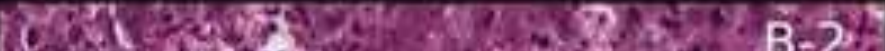
II.

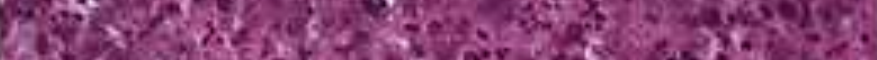

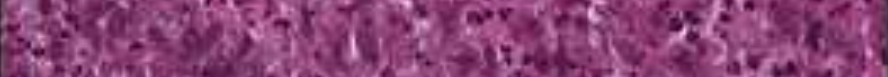

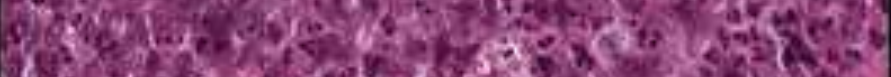

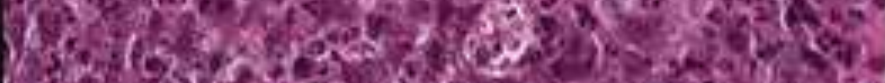

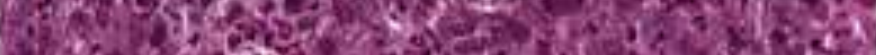

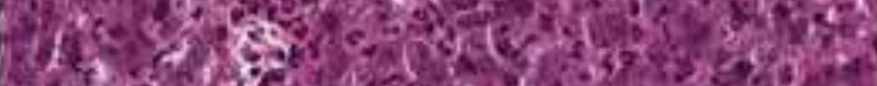

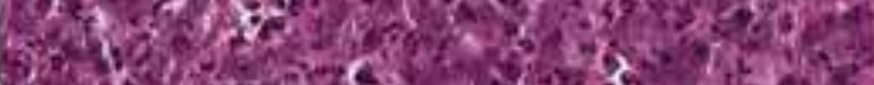

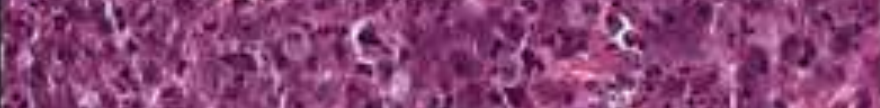

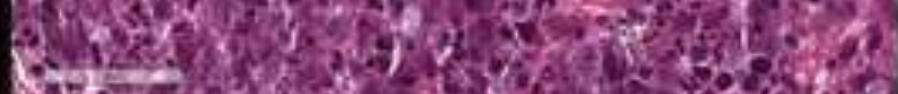

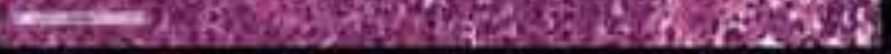

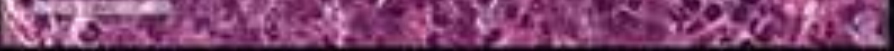
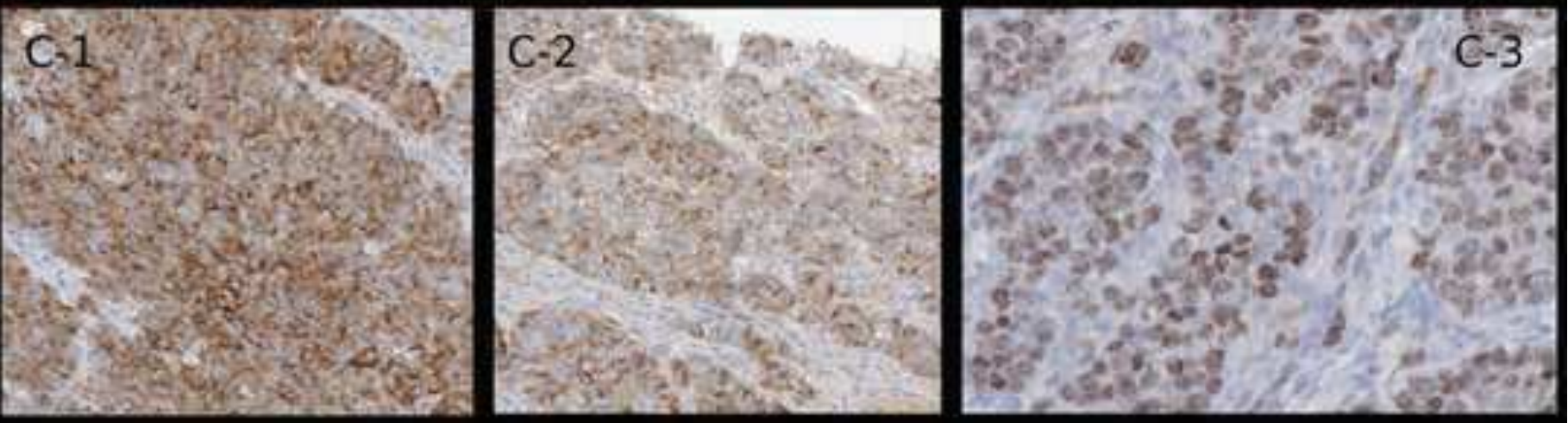


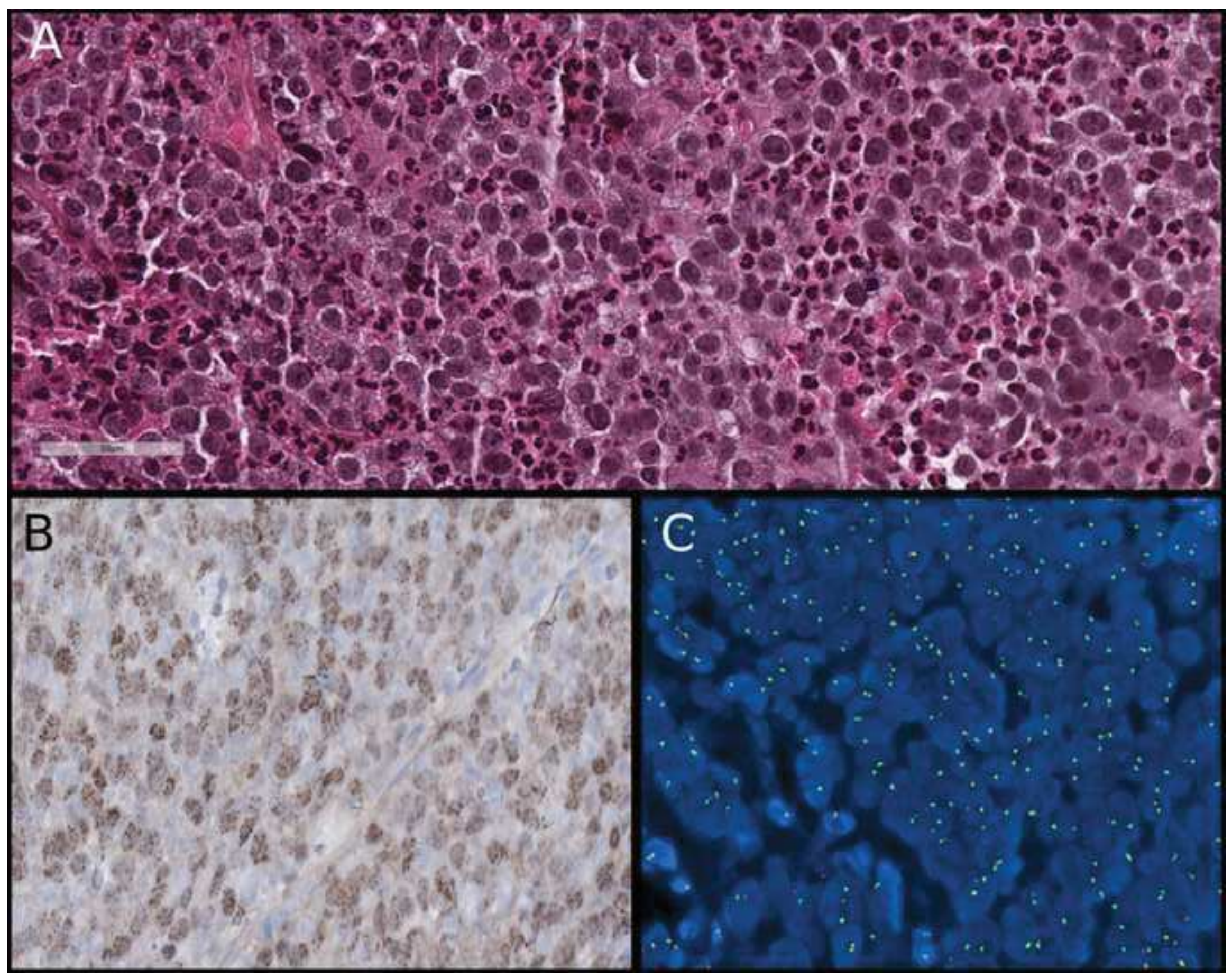

